\title{
COMPORTAMIENTO DE CLONES DE Eucalyptus grandis DEL INTA EN PLANTACIONES EN VERTISOLES DEL CENTRO-SUR DE LA PROVINCIA DE CORRIENTES. ARGENTINA
}

\author{
López, Augusto Javier ${ }^{7}$
}

\section{RESUMEN}

En la República Argentina la región Mesopotámica es la mayor cuenca forestal del país, concentrando aproximadamente el $85 \%$ del total de la superficie forestada. Alrededor del $25 \%$ de la misma corresponde al cultivo con eucaliptos, mayoritariamente Eucalyptus grandis.

Debido al crecimiento sostenido en el ritmo de plantación observado desde finales de la década del 90 se incrementó la utilización de terrenos considerados como de mediana y baja aptitud forestal. El objetivo de este trabajo fue evaluar el comportamiento de 7 clones de 1은 generación del Programa de Mejoramiento genético del INTA en forestaciones comerciales establecidas en Vertisoles del Centro-Sur de la Provincia de Corrientes (29은 28 LS, $58^{\circ} 36^{\prime}$ LW y 83 msnm).

El estudio se realizó en una plantación clonal de 30 meses de edad con preparación del terreno intensiva (subsolado, rastra y camellones), fertilización inicial de $120 \mathrm{~g}$ de fosfato diamónico por planta y una densidad inicial de 833 árboles por hectárea. En la misma se ubicaron parcelas representativas de $240 \mathrm{~m}^{2}$ en las que se midió altura total y diámetro a 1,30 m de altura (DAP) en la totalidad de los individuos existentes en la parcela.

Entre los materiales de mayor crecimiento volumétrico se destacaron los clones EG INTA-157 y EG INTA-36. Las diferencias entre el mejor y peor de los 7 materiales evaluados fue $2,4 \mathrm{~cm}$ en DAP (30,3\%), $2,2 \mathrm{~m}$ en altura $(27,8 \%)$ y $20,7 \mathrm{dm}^{3}$ en volumen medio individual (104\%).

En este tipo de sitios poco explorados para el cultivo de eucalipto en la región y considerados de baja aptitud forestal, se pudieron constatar buenos crecimientos iniciales debido a la calidad genética de los materiales evaluados y a la utilización de esquemas comerciales con buena preparación del terreno y fertilización inicial.

Los crecimientos observados fueron comparables a los obtenidos en otros sitios comúnmente utilizados en las Provincias de Corrientes y Entre Ríos (arenosos profundos y medianamente profundos) y considerados de mayor aptitud productiva para el cultivo de $E$. grandis. La inclusión de dichos suelos permitiría ampliar significativamente la superficie de aptitud forestal en esta región.

Palabras clave: Eucalyptus grandis, Clones, Vertisoles.

${ }^{7}$ Consultor Proyecto PMSRN BIRF 7520 AR. Bella Vista. Corrientes. jal176@hotmail.com 


\section{ABSTRACT}

In the Argentina Republic, the Mesopotamian region is the largest forest basin in the country, concentrating about the $85 \%$ of the total forested area. Around $25 \%$ of it corresponds to Eucalypts, mainly Eucalyptus grandis.

Due to the sustained increase in the plantation rhythm observed since the end of the 90's decade increased also the utilization of land considered as medium and low forest aptitude. The objective of this study was to evaluate the performance of 7 first generation clones from de INTA's Genetic Improvement Program, in forest plantations established in Vertisols in the central south of the province of Corrientes.(29ㅇ $28 \mathrm{SL}, 58^{\circ} 36^{\prime} \mathrm{WL}$ and 83 masl).

The study was conducted in a 30 months old clonal plantation established with intensive soil preparation, $120 \mathrm{~g}$ diamonic phosphate per plant initial fertilization and 833 trees per hectare initial density. In the plantation, $240 \mathrm{~m}^{2}$ representative plots were arranged, and height and diameter at $1.30 \mathrm{~m}$ (DAP) in all samples existing in the plots were measured.

Among the higher volumetric growth materials clones EG INTA-157 and EG INTA-36 were highlighted. The difference between the best and the worst of the 7 evaluated materials was $2,4 \mathrm{~cm}$ in diameter $(30,3 \%), 2,2 \mathrm{~m}$ in height $(27,8 \%)$ and $20,7 \mathrm{dm}^{3}$ in individual average volume $(104 \%)$.

In these kinds of less known sites for eucalypts cultivation, considered as low forest aptitude, it was found good initial growing due to the genetic quality of the evaluated materials and the use of commercial schemes with good soil preparation and initial fertilization.

The observed growths were comparable to those obtained in other commonly used places in the Corrientes and Entre Rios provinces (deep and moderately deep sandy soils) considered as greater productive aptitude for $E$. grandis cultivation. The inclusion of such kind of soils would significantly expand the forest aptitude surface of this area.

Key words: Eucalyptus grandis, Clones, Vertisols. 


\section{INTRODUCCIÓN}

En la República Argentina la región Mesopotámica es la mayor cuenca forestal del País, concentrando aproximadamente el $85 \%$ del total de la superficie forestada. Las forestaciones en dicha región se han desarrollado principalmente en dos áreas consolidadas; Misiones-NE de Corrientes y SE de Corrientes-NE de Entre Ríos; y una que en los últimos años viene rápidamente consolidándose; Centro-SO de la Provincia de Corrientes.

Del total de la superficie forestada en la Mesopotamia, aproximadamente el $25 \%$ corresponde al cultivo de eucaliptos, mayoritariamente Eucalyptus grandis (Braier, 2004). Los tipos de suelos preponderantemente elegidos para forestar con eucaliptos y considerados de gran aptitud forestal han sido aquellos profundos sin problemas de anegamiento (INTA, 1995a; Dalla Tea, 1995). De estos se cuenta con bastante información local (Aparicio y López, 1995; Dalla Tea 1995b; Fernández et al., 1996; Ligier et al., 2000 y López, 2011).

No obstante, debido al crecimiento sostenido del ritmo de plantación desde finales de la década del 90 se incrementó la utilización de terrenos considerados como de mediana y baja aptitud forestal. En este sentido, en la Provincia de Corrientes, se ha comenzado a forestar con eucalipto en la zona del Centro-Sur, representada por los departamentos Curuzú Cuatiá y Sauce.

Esos departamentos ocupan una superficie de 1.160 .600 hectáreas, de las cuales 415.500 ha $(35,8 \%)$ corresponden a Vertisoles y Molisoles del subgrupo Vérticos (Cumba et al., 2005). Este grupo de suelos, de textura fina y típicamente afectados por procesos de contracciónexpansión, cuentan con un gran número de suelos clasificados como de moderada y baja aptitud para el cultivo de eucalipto.

La creciente aplicación de técnicas silvícolas intensivas (preparación del terreno, control de malezas, fertilización) y la utilización de materiales mejorados ha modificado el panorama de terrenos en los cuales no se creía posible forestar o bien o esto era posible con resultados inciertos. Sin embargo, la información disponible sobre sitios de esas características es escasa.

Por lo antes comentado, este trabajo tiene por objetivo evaluar el comportamiento de algunos clones de $1^{\circ}$ generación del Programa de Mejoramiento Genético (PMG) del INTA en forestaciones comerciales establecidas en Vertisoles del Centro-Sur de la Provincia de Corrientes.

\section{MATERIAL Y MÉTODO}

Las evaluaciones se realizaron en una plantación comercial instalada en la primavera del año 2009, ubicada 15 kilómetros al sur de la localidad de Perugorría, Provincia de Corrientes (29ำ 28' LS, $58^{\circ} 36^{\prime}$ LW y 83 msnm).

Los suelos pertenecen al Orden Vertisoles y son similares a los descriptos por el Grupo de Recursos Naturales del INTA Corrientes para las Series Don Luis (Hapludertes típicos) y Don Deolindo (Hapludertes crómicos). Esta última corresponde a la descripción del "lomo" o "cresta" del gilgai, el cual es un microrelieve típico de este tipo de suelos y si bien no ocupa más del $10 \%$ de la superficie se halla relativamente bien distribuido en la misma (Escobar et al., 1996; Cumba et al., 2005).

Estos suelos se encuentran ubicados en medias lomas altas, en un paisaje conformado por lomeríos disectados con pendientes del 2-5\%. En general se encuentran degradados por el sobrepastoreo y se destacan: i) permeabilidad lenta, ii) drenaje moderado a imperfecto, iii) alto riesgo de erosión hídrica y iv) restricciones de orden físico para el desarrollo de raíces en el horizonte subsuperficial.

A efectos de tener información local se escogieron tres lugares representativos del área 
de estudio para extraer muestras y efectuar los análisis químicos. Los resultados se consignan en el Cuadro $\mathrm{N}^{\circ} 1$.

Cuadro $\mathrm{N}^{\circ} 1$

RESULTADOS DE LOS ANÁLISIS DE SUELO DEL ÁREA DE ESTUDIO

\begin{tabular}{|c|c|c|c|c|c|c|c|c|c|}
\hline \multirow{2}{*}{ Serie de Suelo } & \multirow{2}{*}{$\begin{array}{c}\text { Profundidad de } \\
\text { Muestreo } \\
\text { (cm) }\end{array}$} & \multirow{2}{*}{$\mathrm{pH}$} & \multirow{2}{*}{$\underset{(p p m)}{P}$} & $\mathrm{Ca}$ & $\mathrm{Mg}$ & $\mathbf{K}$ & $\mathrm{Na}$ & \multirow{2}{*}{$\begin{array}{l}\text { M.O } \\
\text { (\%) }\end{array}$} & \multirow{2}{*}{$\begin{array}{c}\text { Conductividad } \\
\text { (Mmho/cm) }\end{array}$} \\
\hline & & & & \multicolumn{4}{|c|}{$(\mathrm{cmol} / \mathrm{Kg})$} & & \\
\hline \multirow{2}{*}{ Don Luís } & $0-20$ & 5,50 & 3,0 & 10,5 & 1,5 & 0,11 & 0,54 & 4,8 & 0,07 \\
\hline & $20-40$ & 6,60 & 2,1 & 12,9 & 3,6 & 0,18 & 1,04 & 2,0 & 0,10 \\
\hline \multirow{2}{*}{ Don Luís } & $0-20$ & 5,70 & 2,2 & 10,6 & 2,4 & 0,09 & 0,28 & 4,1 & 0,11 \\
\hline & $20-40$ & 6,40 & 2,2 & 12,6 & 3,4 & 0,10 & 0,49 & 4,0 & 0,07 \\
\hline \multirow{2}{*}{ Don Deolindo } & $0-20$ & 7,08 & 4,3 & 14,6 & 2,4 & 0,20 & 0,44 & 4,7 & 0,22 \\
\hline & $20-40$ & 8,30 & 1,9 & 20,8 & 1,8 & 0,10 & 0,22 & 1,9 & 0,17 \\
\hline
\end{tabular}

El material genético evaluado correspondió a 7 clones de 1ํgeneración del PMG del INTA. Estos materiales se encuentran inscriptos en el Registro Nacional de Cultivares (RNC) del Instituto Nacional de Semillas (INASE) y son de uso público.

1) y consistió en:

La preparación del terreno fue intensiva sobre la banda de plantación (Figura $\mathrm{N}^{\circ}$

Subsolado de 30 a $40 \mathrm{~cm}$ de profundidad

Dos pasadas de rastra en la banda de plantación

Formación de un camellón de $120 \mathrm{~cm}$ de ancho y $40 \mathrm{~cm}$ de alto.
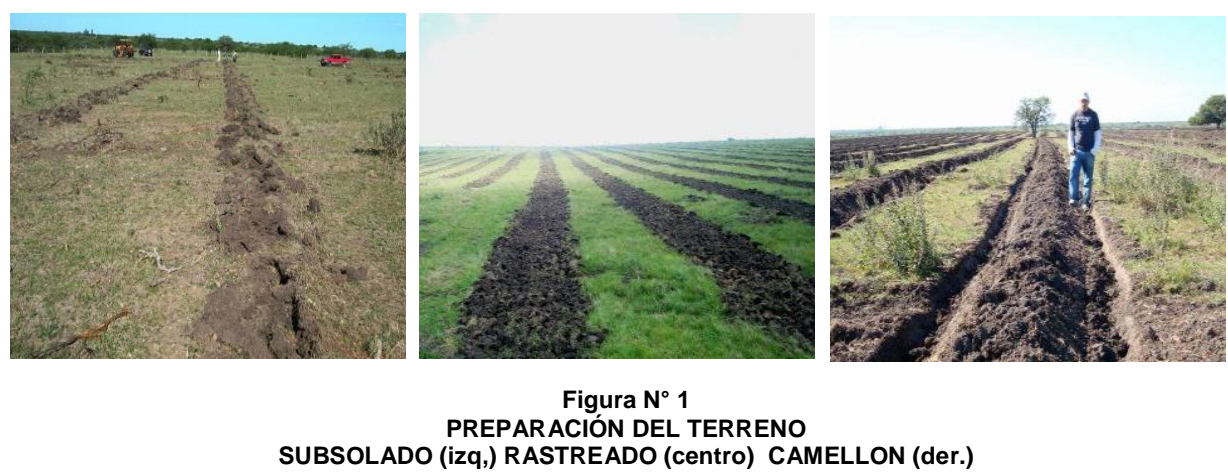

La densidad de plantación fue de 833 plantas/ha $(4 \times 3 \mathrm{~m})$. El control de malezas se realizó con herbicidas pre-emergentes y post-emergentes solo en la banda de plantación $(37,5 \%$ de la dosis agrícola por hectárea).

Las mediciones de crecimiento se realizaron a la edad de 30 meses. Para ello se instalaron 35 parcelas temporarias representativas de los sitios forestados (5 parcelas/clon). Las mismas contaron con una superficie de $240 \mathrm{~m} 2$ y 25 a 30 individuos por parcela. En cada parcela 
se midió altura total (ALT) utilizando un VERTEX II@ $(10 \mathrm{~cm}$ precisión) y diámetro a 1,3 metros (DAP) con Forcípula Haglöf® (1 mm de precisión) en la totalidad de los individuos existentes en la parcela.

Friedl (1988):

El volumen individual (VOL) se calculó utilizando la ecuación propuesta por Glade y

$$
\text { VOL }=\exp \left[-9,9616718+\left(1,82344264^{*} \operatorname{Ln} \text { Diámetro a 1,30) +(1,0697836* Ln Altura }\right)\right]
$$

Con la altura, el diámetro y el volumen individual se calculó el volumen por hectárea (Volha) y los Incrementos Medios Anuales (IMA) de la altura (IMA ALT), el diámetro (IMA DAP) y el Volha (IMA Volha).

\section{RESULTADOS Y DISCUSIÓN}

En el Cuadro $\mathrm{N}^{\circ} 2$ se presentan los valores medios y desvíos entre parcelas de cada uno de los clones evaluados. Las diferencias entre el mejor y peor de los 7 materiales fue $2,4 \mathrm{~cm}$ en DAP, $2,2 \mathrm{~m}$ en ALT y $20,7 \mathrm{dm}^{3}$ en VOL, lo que equivale a una diferencia de $30,3 \%, 27,8 \%$ y $104 \%$, respectivamente. Los materiales de mayor crecimiento volumétrico fueron los clones EG INTA-157 y EG INTA-36 (Figura $N^{\circ} 2$ ). Estos materiales mostraron los mayores crecimientos tanto en ALT como en DAP.

\section{Cuadro $\mathrm{N}^{\circ} 2$}

VALORES MEDIOS, DESVIOS ENTRE PARCELAS E IMA DE LOS CLONES EVALUADOS

\begin{tabular}{|c|c|c|c|c|c|c|c|c|c|c|}
\hline CLON & & $\begin{array}{l}\text { LT } \\
\text { desv }\end{array}$ & $\begin{array}{c}\mathrm{D}^{\prime} \\
(\mathrm{cm})\end{array}$ & $\begin{array}{l}P \\
\text { desv }\end{array}$ & $\begin{array}{r}\text { VC } \\
\left(\mathrm{dm}^{3}\right)\end{array}$ & desv & $\begin{array}{c}\text { Volha } \\
\left(\mathrm{m}^{3} \cdot \mathrm{ha}^{-1}\right)\end{array}$ & $\begin{array}{l}\text { IMA DAP } \\
\text { (cm.año-1) }\end{array}$ & $\begin{array}{l}\text { IMA ALT } \\
\text { (m.año-1) }\end{array}$ & $\begin{array}{l}\text { IMA Volha } \\
\left(\mathrm{m}^{3} \cdot \mathrm{ha}^{-1} \cdot \mathrm{año}^{-1}\right)\end{array}$ \\
\hline EG INTA-1 & 10,0 & $(0,5)$ & 9,1 & $(0,5)$ & 31,4 & $(4,4)$ & 26,1 & 3,63 & 3,98 & 10,45 \\
\hline EG INTA-2 & 7,9 & $(1,0)$ & 7,9 & $(1,2)$ & 19,9 & $(7,1)$ & 16,6 & 3,17 & 3,15 & 6,62 \\
\hline EG INTA-35 & 9,5 & $(0,6)$ & 9,1 & $(0,6)$ & 30,4 & $(6,0)$ & 25,3 & 3,66 & 3,81 & 10,12 \\
\hline EG INTA-36 & 10,3 & $(0,8)$ & 9,5 & $(0,8)$ & 35,4 & $(7,7)$ & 29,5 & 3,80 & 4,11 & 11,81 \\
\hline EG INTA-152 & 9,4 & $(0,7)$ & 9,0 & $(0,7)$ & 28,9 & $(6,1)$ & 24,1 & 3,59 & 3,75 & 9,62 \\
\hline EG INTA-157 & 10,1 & $(0,9)$ & 10,3 & $(0,9)$ & 40,5 & $(10,1)$ & 33,8 & 4,13 & 4,02 & 13,50 \\
\hline EG INTA-164 & 8,7 & $(0,6)$ & 8,6 & $(0,6)$ & 24,8 & $(4,9)$ & 20,7 & 3,43 & 3,47 & 8,27 \\
\hline
\end{tabular}

Los clones EG INTA-164 y EG INTA-2 fueron los de menor crecimiento volumétrico. Dichos clones no evidenciaron un buen comportamiento en estos sitios. Sin embargo, ambos clones tuvieron buenos comportamientos en otros suelos de Corrientes, estando ubicados entre los 3 de mayores crecimientos en DAP de 11 clones evaluados y el clon EG INTA-2 también entre los 3 primeros en ALT (Aparicio, 2012).

Por su parte, los clones EG INTA-1, EG INTA-35 y EG INTA-152 mostraron crecimientos intermedios y similares entre sí. Los VOL de estos materiales variaron de $28,9 \mathrm{dm}^{3}$ a $31,4 \mathrm{dm}^{3}$ y manifestaron poca variación entre parcelas.

El Incremento Medio Anual de la altura de los clones evaluados varió de 3,15 a 4,11 $\mathrm{m} / \mathrm{año}$, siendo la media de los 7 clones evaluados 3,76 m/año. 

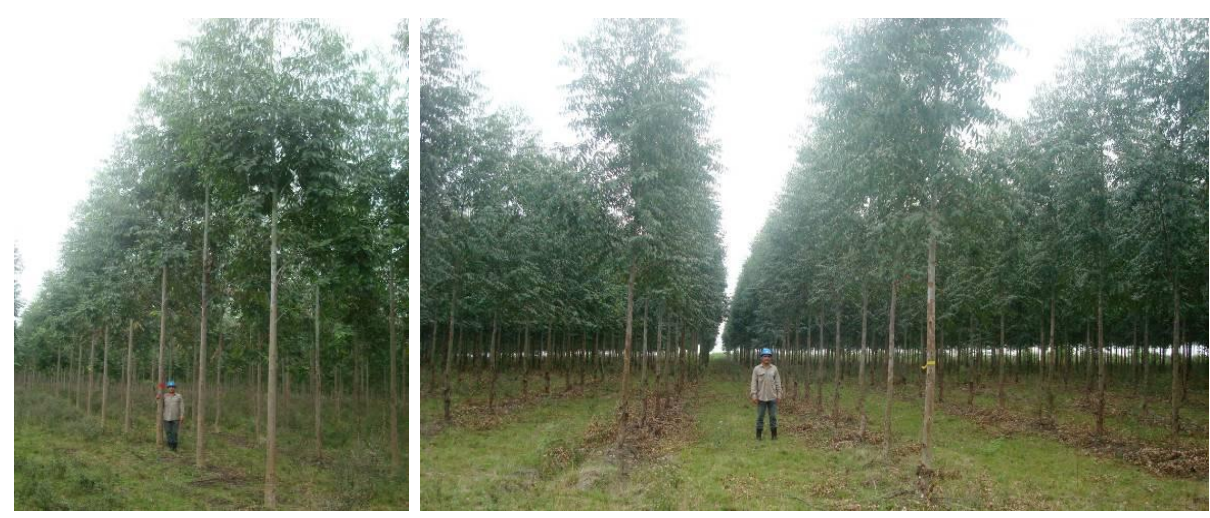

Figura $\mathbf{N}^{\circ} 2$

ESTADO DE LAS PANTACIONES A LOS 30 MESES DE EDAD

CLON EG INTA 157 (izq.) CLON EG INTA 36 (der.)

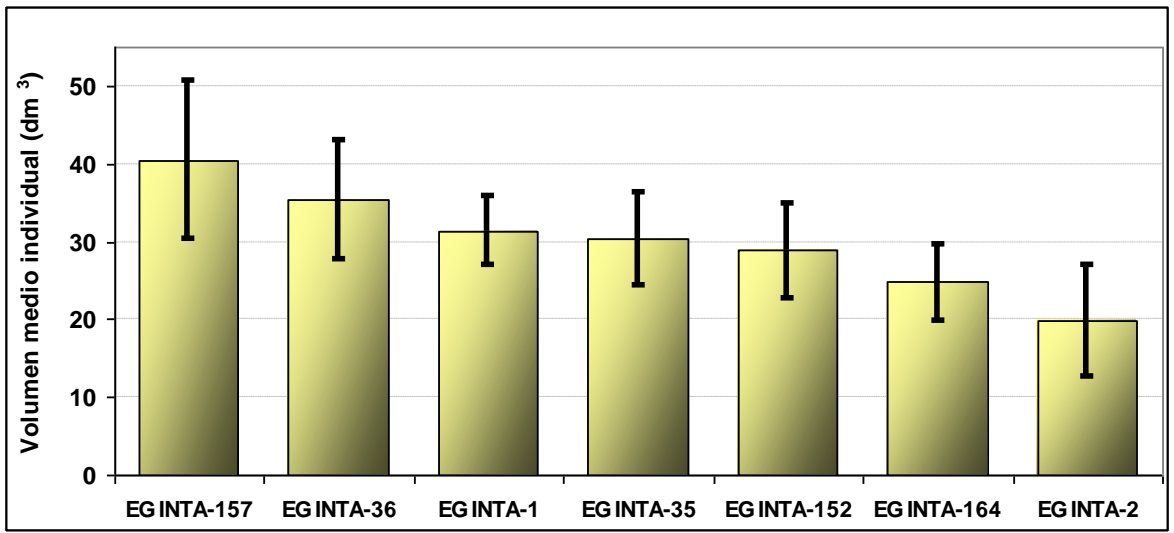

Figura $\mathrm{N}^{\circ} 3$

VOLUMEN INDIVIDUAL A LOS 30 MESES DE EDAD Y DESVÍOS ENTRE PARCELAS

DE LOS 7 CLONES EVALUADOS

En el Cuadro $\mathrm{N}^{\circ} 3$ se muestran valores de crecimiento obtenidos a edades similares en otras experiencias realizadas en la región (como variable de comparación se consideró IMA ALT.

Como se observa en el cuadro, el IMA ALT informado por Aparicio (2012) en suelos arenosos hidromórficos medianamente profundos fue de 3,5 m/año, el cual es similar al IMA ALT promedio de los 7 clones evaluados en el presente trabajo.

Así mismo, considerando los 3 clones de mejor crecimiento los valores son comparables a los obtenidos por López (1991 y 1998) para suelos arenosos profundos y medianamente profundos del NO de la Provincia de Corrientes. 
Cuadro $\mathrm{N}^{\circ} 3$

INCREMENTOS MEDIOS ANUALES EN LA ALTURA OBTENIDOS

EN OTRAS EXPERIENCIAS EN LA REGIÓN

\begin{tabular}{|c|c|c|c|c|c|}
\hline Zona & $\begin{array}{c}\text { Edad } \\
\text { Evaluación } \\
\text { (meses) }\end{array}$ & Material Genético & Suelo & $\begin{array}{l}\text { IMA ALT } \\
\left(\mathrm{m} \cdot a \tilde{0} 0^{-1}\right)\end{array}$ & Referencia \\
\hline \begin{tabular}{|l} 
Noreste \\
Corrientes
\end{tabular} & 24 & $\begin{array}{l}\text { Clones comerciales de } \\
\text { INTA (1) }\end{array}$ & Arcilloso rojo profundo & 5,03 & CFCN (2009) \\
\hline \multirow{2}{*}{$\begin{array}{l}\text { Oeste } \\
\text { Corrientes }\end{array}$} & \multirow{2}{*}{22} & \multirow{2}{*}{$\begin{array}{l}\text { Progenies de polinización } \\
\text { libre de } 9 \text { individuos } \\
\text { selectos de materia local }\end{array}$} & Arenoso profundo & 5,69 & \multirow{2}{*}{ López (1991) } \\
\hline & & & $\begin{array}{l}\text { Arenoso medianamente } \\
\text { profundo }\end{array}$ & 4,06 & \\
\hline \begin{tabular}{|l} 
Oeste \\
Corrientes
\end{tabular} & 24 & $\begin{array}{l}\text { Clones experimentales de } \\
\text { INTA }\end{array}$ & $\begin{array}{l}\text { Arenoso profundo } \\
\text { (Alfisoles) }\end{array}$ & 4,15 & López (1998) \\
\hline $\begin{array}{l}\text { Centro-Este } \\
\text { Corrientes }\end{array}$ & 30 & $\begin{array}{l}\text { Clones comerciales de } \\
\text { INTA (2) }\end{array}$ & $\begin{array}{l}\text { Arenoso hidromórfico } \\
\text { Medianamente profundo } \\
\text { (Entisoles) }\end{array}$ & 3,50 & Aparicio (2012) \\
\hline $\begin{array}{l}\text { Noreste } \\
\text { Entre Ríos }\end{array}$ & 54 & Semilla comercial (3) & $\begin{array}{l}\text { Arcilloso negro } \\
\text { (Vertisoles) }\end{array}$ & 3,03 & Marcó (1983) \\
\hline \begin{tabular}{|l|} 
Centro \\
Entre Ríos
\end{tabular} & 17 & $\begin{array}{l}\text { Material de semilla } \\
\text { E. grandis (4) }\end{array}$ & $\begin{array}{l}\text { Arcilloso negro } \\
\text { (Vertisoles-Molisoles) }\end{array}$ & 2,56 & $\begin{array}{l}\text { Larocca } \\
\text { et al. (2001) }\end{array}$ \\
\hline
\end{tabular}

(1) Promedio de los Clones EG INTA-2, EG INTA-35, EG INTA-157 y EG INTA-164.

(2) Promedio de los Clones EG INTA-1, EG INTA-2, EG INTA-35, EG INTA-36, EG INTA-157 y EG INTA-164.

(3) Huerto semillero Sudáfrica

(4) Semilla de procedencia sudafricana (Pambult)

En cuanto a suelos similares a los descriptos en este trabajo, Marcó (1983) y Larocca et al. (2001) citan para plantaciones ubicadas en Vertisoles de la Provincia de Entre Ríos valores de crecimiento de 3,03 y 2,56 metros/año respectivamente. Estos incrementos son 32 y $19 \%$ inferiores a los obtenidos en esta evaluación.

En este tipo de sitios poco explorados para el cultivo de eucalipto en la región, considerados de baja aptitud, se pudo constatar buenos crecimientos iniciales de los materiales evaluados debido a su calidad genética y a la utilización de prácticas de preparación del terreno adecuadas, control de malezas y fertilización inicial incluidas en estos esquemas comerciales.

Los crecimientos observados fueron comparables a los obtenidos en otros sitios comúnmente utilizados en las Provincias de Corrientes y Entre Ríos (arenosos profundos y medianamente profundos) y considerados de mayor aptitud productiva para el cultivo de $E$. grandis. La inclusión de dichos suelos acompañada de la tecnología adecuada permitirá ampliar significativamente la superficie de aptitud en esta región.

En base a los resultados presentados se infiere que la aptitud edáfica de estos sitios no sería limitante para el cultivo de eucaliptos. No obstante, en los últimos años las bajas temperaturas invernales comenzaron más fuertemente a ser una limitante para el desarrollo del cultivo en esta zona.

Debido al paisaje fuertemente disectado con abundantes cañadas y depresiones que transportan y acopian el aire frío en algunos sectores, resulta de gran importancia la elección de los sitios de plantación evitando bajos, depresiones y cualquier sector donde se acumule el aire frío. De igual modo, diseños de las plantaciones que permitan el drenaje del aire frío hacia zonas no forestadas, las fertilizaciones con Potasio y el control de las plantas competidoras reducirían la 
incidencia de la heladas (Larocca, 2012).

Si bien lo antes mencionado contribuye a disminuir el efecto e incidencia de las heladas, es necesaria la inclusión de materiales de mayor tolerancia a las bajas temperaturas, por lo menos en parte de los sistemas productivos de esta región. En este sentido el INTA cuenta con clones de híbridos interespecíficos de E. grandis $\times$ E. camaldulensis, E. grandis $\times$ E. teriticornis y E. grandis $\mathrm{x}$ E. dunnii, los cuales han mostrado crecimientos similares a $E$. grandis y mayor tolerancia al frío (Marco y Harrand, 2012). Seis de estos clones ya se encuentran inscriptos en el RNC del INASE y en breve estarán disponibles para el sector productivo.

\section{CONCLUSIONES}

Se constataron buenos crecimientos iniciales de los materiales evaluados, siendo los clones EG INTA-157 y EG INTA-36 los materiales de mayor crecimiento volumétrico. La diferencia entre el mejor y el peor de los 7 materiales evaluados fue de $104 \%$ en volumen medio individual.

Los crecimientos observados fueron comparables a los obtenidos en otros sitios comúnmente utilizados en la región considerados de mayor aptitud productiva para el cultivo de $E$. grandis.

La inclusión de estos suelos acompañada de la tecnología adecuada permitirá ampliar significativamente la superficie de aptitud en esta región, no obstante, es necesaria la inclusión de materiales resistentes a frío en estos sistemas productivos.

\section{REFERENCIAS}

Aparicio, J. L. y López, J. A., 1995. Potencial de Eucalyptus grandis en los suelos del sudeste de la provincia de Corrientes y algunos factores edáficos relacionados con la producción de madera. Bosque 16(2): 81-98. Disponible en: http://mingaonline.uach.cl/pdf/bosque/v16n2/art07.pdf

Aparicio, J. L., 2012. Comportamiento de clones de Eucalyptus grandis en relación a un material de semilla en un suelo arenoso de Corrientes. XXVI Jornadas Forestales de Entre Ríos. 4 y 5 de Octubre de 2012.

Braier, G., 2004. Tendencias y perspectivas del sector forestal al año 2020 Argentina. SAGPyA-FAO. Dirección de Forestación: $71 \mathrm{p}$

CFCN, 2009. Consorcio Forestal Corrientes Norte. 2009. Reunión Nro. 179. Gobernador Virasoro. Corrientes.

Cumba, A.; Imbellone, P. y Ligier, D., 2005. Propiedades morfológicas, físicas, químicas y mineralógicas de suelos del sur de Corrientes. Revista de la Asociación Geológica Argentina 60 (3): 571-582. Disponible en: http://www.scielo.org.ar/pdf/raga/v60n3a13.pdf

Dalla Tea, F., 1995a. Efecto de la densidad de plantación sobre el crecimiento de Eucalyptus grandis en Entre Ríos, Argentina. Invest. Agr.: Sist. Recur. For. Vol. 4 (1). Pag: 57-71.

Dalla Tea, F., 1995b. Factores del suelo que afectan la productividad del Eucalypus grandis. Disponible en: http://www.inta.gov.ar/concordia/info/indices/tematica/cd-informacionforestal/C9.pdf

Escobar, E. H.; Ligier, D. L.; Melgar, R.; Matteio, H. y Vallejos, O., 1996. Mapa de Suelos de la Provincia de Corrientes 1:500.000. Área de Producción Vegetal y Recursos Naturales E.E.A. INTA-Corrientes. 432 p.

Fernández, R. A.; Pahr, N. M.; Lupi, A. M. y Fassola, H. E., 1996. Evaluación del crecimiento de Eucalyptus grandis Hill. Ex Maiden en diferentes condiciones de sitio en el nordeste argentino. ProFoMe (Proyecto Integrado Forestal Mesopotámico). EEA Montecarlo. Centro Regional Misiones. ISSN-0327-926. 6 p. Disponible en:http://www.inta.gov.ar/montecarlo/INFO/documentos/forestales/3_Evaluacion_crecimiento_Eucalyptus_grandis .pdf

Glade, J. E. y Friedl, R. A., 1988. Ecuaciones de volumen para Eucalyptus grandis Hill ex Maiden en el Noreste 
de Entre Ríos. En: VI Congreso Forestal Argentino. Santiago del Estero. Actas. Tomo II: 416-420.

Ligier, H. D.; Gimenez, L.; Kurtz, D.; López, J. y Perucca, R., 2000. Relaciones entre Índice de Crecimiento Medio Anual y propiedades de los suelos en montes forestales de Eucalyptus grandis y Pinus elliottii en la cuenca del Río Uruguay. Corrientes. Argentina. UNNE. Comunicaciones Científicas y Tecnológicas: 4 p. Disponible en: http://www1.unne.edu.ar/cyt/2000/5_agrarias/a_pdf/a_069.pdf

Larocca, F.; Marcó, M. A. y Rosembereg, J., 2001. Evaluación de las posibilidades de desarrollo forestal de los suelos del área ganadera del Norte de Entre Ríos. PIA 33/97

Larocca, F., 2012. Daños por heladas en plantaciones de Eucalyptus grandis. Posibles herramientas desde el manejo. XXVI Jornadas Forestales de Entre Ríos. 4 y 5 de Octubre. 10 p.

López, A. J., 1991. Evaluación del crecimiento y estimación de Parámetros Genéticos en progenies de Eucalytus grandis de procedencia local. En: Jornadas de Eucaliptos de alta productividad. Centro de Investigaciones y Experiencias Forestales (CIEF). 6-7 de Diciembre de 1991. Pag: 118-127.

López, A. J., 1998. Comportamiento de 12 clones de Eucalytus grandis al segundo año de crecimiento. II Jornada Forestal. Estación experimental agropecuaria Bella Vista. Pag: 23-30.

López, A. J., 2011. Eucalyptus grandis en el Sudeste de Corrientes: Propiedades físicas y químicas del suelo relacionadas con la producción volumétrica. XXV Jornadas Forestales de Entre Ríos. 20 y 21 Octubre de 2011. $7 \mathrm{p}$.

INTA, 1995. Manual para Productores de Eucaliptos de la Mesopotamia Argentina. Instituto Nacional de Tecnología Agropecuaria. ISBN 950-9853-55-0. ISBN 950-9853-55-0. Editor: Grupo Forestal, EEA. INTA. Concordia. C.C N 34, (3200) Concordia, Entre Ríos, Argentina. Pag: 22-26.

Marcó, M. A., 1983. Comportamiento del E. grandis local en relación a procedencias introducidas de Sudáfrica. Resultados a los 4,5 años. ISSN 0326-5005. 4 p.

Marcó, M. A. y Harrand, L., 2012. El Programa de Mejora de Eucaliptos del INTA en La Mesopotamia Argentina. En: Resúmenes Jornadas de Actualización Técnica. Mejoramiento Genético de Pinos y Eucaliptos Subtropicales. $1^{\text {a }}$ edición. ISBN 978-978-679-144-1. Pag 44-48. 
